\section{Lipomatous hypertrophy of the interatrial septum}

A

69 year old woman with a history of obesity, hypertension, nicotine abuse, and periods of atrial flutter, was admitted with a New York Heart Association (NYHA) grade III dyspnoea. She did not complain of angina. Chest $x$ ray revealed no signs of cardiomegaly or pulmonary oedema. The ECG showed a sinus rhythm with premature atrial complexes. Transoesophageal echocardiography (TOE) showed a large mass located in the right atrium (RA) extending along the atrial septal wall and compressing the inferior caval vein. There was a grade I/IV tricuspid insufficiency. Nuclear magnetic resonance imaging revealed a right atrial mass (maximal diameter of $5.5 \mathrm{~cm}$ ) originating from the atrial septum, which was suspected as being a myxoma or lipoma. Coronary angiography showed no signs of coronary disease. In December 1999 she underwent an operation. After median sternotomy, bicaval cannulation and hypothermia $\left(32^{\circ} \mathrm{C}\right)$ were used for cardiopulmonary bypass. After opening the right atrium, a mass was visible originating from the atrial septum with a maximal diameter in the area of the superior caval vein $(6 \mathrm{~cm})$. By opening the foramen ovale and palpating the left atrium (LA), there appeared to be the same mass on the free left atrial wall, extending to the ostia of the pulmonary veins. Macroscopically it appeared to be lipomatous tissue. Frozen section diagnosis confirmed lipomatous hypertrophy. It was impossible to resect the tumour totally because of its location. There- fore extensive debulking was performed, with a reduction of $4 \mathrm{~cm}$ in diameter (confirmed by TOE). The atriotomy was closed and the patient was weaned successfully from extracorporeal circulation. For 15 days after surgery there were recurrent periods of atrial fibrillation and flutter, which were treated pharmacologically and, on two occasions, with electrical cardioversion. The patient was discharged in good condition and in sinus rhythm.

N Haenen

W Morshuis

R Heijmen

W Jaarsma na_haenen@hotmail.com

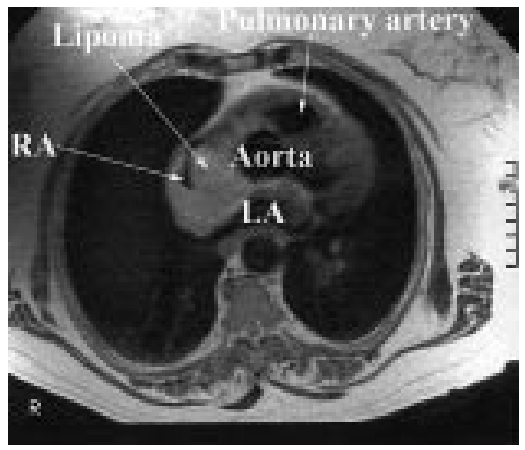

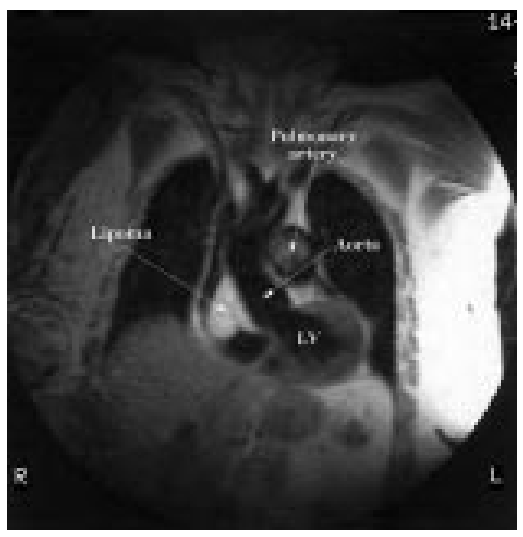

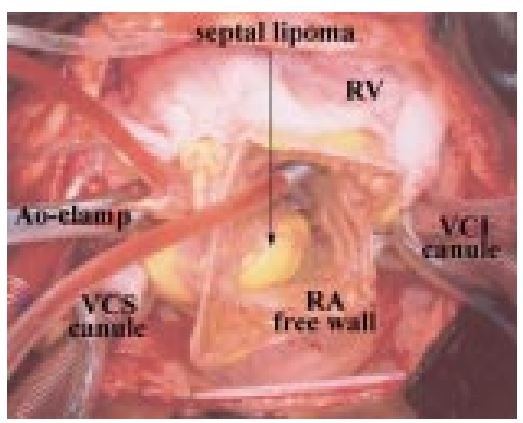

\title{
Imaging of massive pulmonary embolism
}

$A^{2}$ 26 year old woman was admitted with fever and illness caused by an infiltration of the lingula. The day after admittance, symptoms of acute dyspnoea and tachypnoea developed. Pulmonary embolism was suspected. A perfusion scan showed complete absence of perfusion of the right lung with abnormalities in the left lung fitting her extensive lingula infiltration (below left: panel A). Pulmonary angiography was carried out to determine the precise location of the obstruction. This showed a total obstruction directly proximal in the right pulmonary artery (below centre:

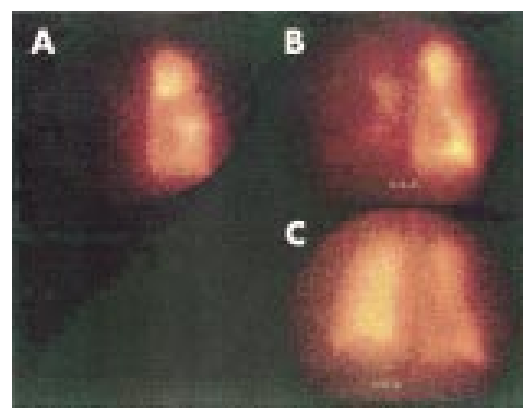

A, superior vena cava; $B$, truncus pulmonalis; C, obstruction). The patient received frontloading recombinant tissue plasminogen activator ( $r$-tPA). Then transoesophageal echocardiography was performed. A massive thrombus was visible with almost complete obstruction of the vessel (below right: A, aorta; B, thrombus)

The next day the patient underwent a controlled ventilation-perfusion scan which still showed a major perfusion defect of the right lung (below left: panel B) during normal ventilation (panel C). Because of threatening respiratory insufficiency, she underwent an

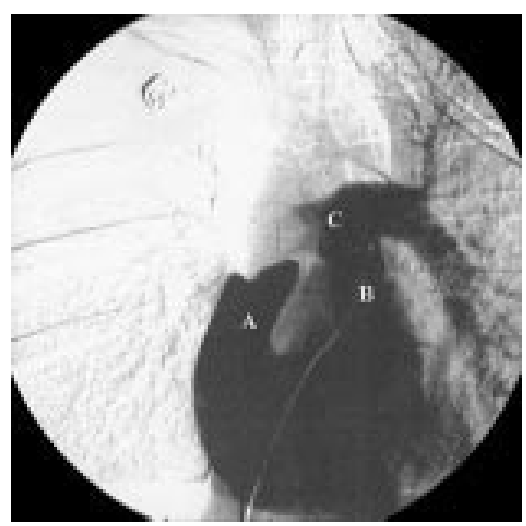

embolectomy. The procedure was completed without complications. Echographic examination of the pelvis and legs showed total thrombosis of the right femoral vein and a thrombus in the wall of the left iliac communis vein. Therefore a vena cava filter was introduced postoperatively. Three weeks after surgery she was discharged from hospital in good health.

N Haenen

D Odekerken

W Jaarsma na_haenen@hotmail.com

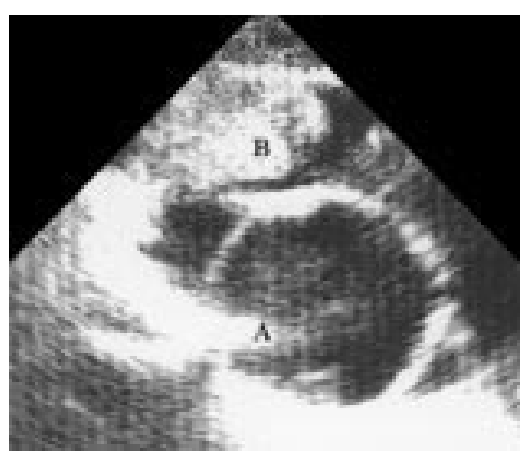

